\title{
IRREDUCIBLE GRAPHS
}

BY J. W. T. YOUNGS

Communicated January 22, 1964

A graph $G$ is $n$-irreducible if it cannot be imbedded in an orientable 2-manifold $M$ of genus $(n-1)$, but, for any arc $a$ in $G$, the graph $G_{a}$, obtained by removing $a$ from $G, c a n$ be imbedded in $M$.

A classical result of Kuratowski states that there are essentially only two 1 -irreducible graphs. These are $K_{5}$, the complete 5 -graph (five vertices and all possible connecting arcs), and $K_{3,3}$, a hexagon with its "long" diagonals.

Practically nothing is known about $n$-irreducible graphs for $n>1$. For example, it is not even known whether the collection of 2-irreducible graphs is finite. (See $[2$, p. 63].)

Under the circumstances it is interesting to observe that $n$-irreducible graphs for $n>1$ can be constructed easily as a consequence of one of the principal results in [1].

In order to conserve space the reader is referred to [3] for basic definitions and background information.

The first result is a useful and simple theorem characterizing $n$-irreducibility.

Theorem. A graph $G$ is n-irreducible if and only if $\gamma(G)=n$ and $\gamma\left(G_{a}\right)=(n-1)$ for any arc $a$ in $G$.

Proof. If $G$ is $n$-irreducible, then, by definition,

$$
\begin{aligned}
\gamma(G) & \geqq n, \\
\gamma\left(G_{a}\right) & \leqq(n-1) .
\end{aligned}
$$

Suppose $M$ is an orientable 2-manifold of genus $\gamma\left(G_{a}\right)$. Then $G_{a}$ can be imbedded in $M$. By adding a suitably located "handle" to $M$ one can accommodate the arc $a$ missing from $G$; in short, imbed $G$. The new 2-manifold has genus one larger than $M$. This fact, together with (1) and (2), implies

$$
n \leqq \gamma(G) \leqq \gamma\left(G_{a}\right)+1 \leqq n .
$$

Consequently equality signs hold throughout, and

$$
\begin{aligned}
\gamma(G) & =n \\
\gamma\left(G_{a}\right) & =(n-1) .
\end{aligned}
$$


Conversely, $\gamma(G)=n$ implies that $G$ cannot be imbedded in an orientable 2-manifold of genus $(n-1)$, while $\gamma\left(G_{a}\right)=(n-1)$ implies that $G_{a}$ can. Hence $G$ is $n$-irreducible.

The main result of $[1]$ referred to above is that if $B^{1}, \cdots, B^{n}$ are the blocks of $G$, then

$$
\gamma(G)=\sum_{1}^{n} \gamma\left(B^{i}\right) .
$$

It is now a simple matter to construct $n$-irreducible graphs in many ways. For example, suppose $B^{1}, \cdots, B^{n}$ are graphs each of which is a copy of $K_{5}$ or $K_{3,3}$. In each $B^{i}$, select a vertex $v_{i}$, and identify $v_{1}, \cdots, v_{n}$. The graph $G=\bigcup_{1}^{n} B^{i}$ is connected and has blocks $B^{1}, \cdots, B^{n}$. Since $\gamma\left(B^{i}\right)=1, i=1, \cdots, n$, an application of (3) yields

$$
\gamma(G)=n .
$$

On the other hand, if any $\operatorname{arc} a$ is removed from $G$, it is removed from some $B^{i}$, say $B^{1}$, which now becomes $B_{a}^{1}$. Thus

$$
G_{a}=B_{a}^{1} \cup \bigcup_{2}^{n} B_{i}
$$

and the $B^{\prime}$ s above are the blocks of $G_{a}$. Since $B^{1}$ is 1 -irreducible, $\gamma\left(B_{a}^{1}\right)=0$. Hence, a second use of (1) gives

$$
\gamma\left(G_{a}\right)=(n-1) .
$$

The theorem now guarantees that $G$ is $n$-irreducible.

A question which suggests itself immediately is this: Are there $n$ irreducible graphs which are not given by the above construction? The answer is in the affirmative. The graphs, however, are by no means easy to construct, nor are their properties transparent. Discourse on the matter is therefore postponed until a time when graphs approaching the beauty of those due to Kuratowski may be discovered, and the accompanying exposition enhanced with some measure of elegance.

\section{BiBLIOGRAPHY}

1. Joseph Battle, Frank Harary, Yukihiro Kodama and J. W. T. Youngs, Additivity of the genus of a graph, Bull. Amer. Math. Soc. 68 (1962), 565-568.

2. G. Ringel, Färbungsprobleme auf Flächen und Graphen, Mathematische Monographien, 2, VEB Deutscher Verlag der Wissenschaften, Berlin, 1959.

3. J. W. T. Youngs, The genus of a graph, J. Math. Mech. 12 (1963), 303-315.

INDIANA UNIVERSITY AND

Institute for Defense Analyses 